\title{
Introductory notes for the Acta IMEKO Special Issue on "Historical papers on fundamentals of measurement"
}

\author{
L. Mari \\ School of Industrial Engineering, Università Carlo Cattaneo - LIUC, C.so Matteotti, 22 - 21053 Castellanza (VA) - Italy
}

IMEKO dedicates this Special Issue to 2014 World Metrology Day, that celebrates the signature of the Metre Convention on May $20^{\text {th }} 1875$ by representatives of seventeen nations

Section: EDITORIAL

Keywords: measurement theory, uncertainty, history

Citation: L. Mari, Introductory notes for the Acta IMEKO Special Issue on "Historical papers on fundamentals of measurement", Acta IMEKO, vol. 3, no. 1, article 2, May 2014, identifier: IMEKO-ACTA-03 (2014)-01-02

Editor: Paolo Carbone, University of Perugia

Received May $1^{\text {st }}$, 2014; In final form May 1 ${ }^{\text {st }}$, 2014; Published May 2014

Copyright: @ 2014 IMEKO. This is an open-access article distributed under the terms of the Creative Commons Attribution 3.0 License, which permits unrestricted use, distribution, and reproduction in any medium, provided the original author and source are credited

Corresponding author: L. Mari, e-mail: Imari@liuc.it

The term "Acta IMEKO" was originally used to designate the proceedings of the IMEKO World Congresses, and explicitly the first twelve Congresses from Budapest, Hungary, 1958, to Beijing, China, 1991, held in a period of about thirty crucial years for our scientific, technological, social, and political history. This Special Issue of the (new) Acta IMEKO journal offers the reader a small selection of papers on fundamentals aspects of measurement, that were originally published in those proceedings. It is an opportunity, first of all, to pay a tribute to the many distinguished colleagues that excellently contributed to the development of measurement science in the context of IMEKO, but also to celebrate IMEKO in its history and unique role in the promotion of international sharing of knowledge and collaboration among scientists.

Despite the common feeling that we live in an epoch of extraordinary, and extraordinarily rapid, changes, the papers that have been reissued here mainly maintain their scientific significance, for the outstanding visions and analyses that propose, while their most temporally situated positions are often of great interest as lively witnesses of what the state and the perspectives of measurement science were a few decades ago. A chronological study on the (original) Acta IMEKO might be conducted, for example, on the changes induced on measurement by the progressive diffusion of digital devices and numerical methods, and the corresponding formation of the concept of measuring systems as information machines.

Unmodified significance of the basic proposals and historical interest of the foreseen perspectives: these have been the guiding principles in the selection of the papers for the present
Special Issue, all of them sharing an interest in contributing to the development of a well-structured, systemic interpretation of the fundamentals of measurement (the selection has been limited by the unfortunate fact that not all volumes of the proceedings were available in the selection stage).

In the present social and economic situation more and more emphasizing the role and the importance of measurement processes, identifying the pivotal elements of a culture of measurement becomes a critical task for metrology, the "science of measurement and its application" (as the International Vocabulary of Metrology defines it), possibly formalized in the development of a body of knowledge on measurement science. The diachronic perspective offered by these papers might give some contributions in highlighting continuities and discontinuities in fundamental concept formation in the last fifty years of measurement-related scientific and technical history.

The reader might be interested to know that the current default choice of English as the only language for the published papers is relatively recent in the context of the IMEKO community: in the first fifteen years of Acta IMEKO, multiple options were available as for the language of papers (and the proceedings of the first two Congresses, Budapest, Hungary, 1958 and 1961, include texts in five languages, English, French, German, Hungarian, and Russian!). On the other hand, only English written papers have been selected for this Special Issue.

The reissued papers are identical in contents to the originals (having thus accepted lack of homogeneity in the layout, abstract and keywords, sometimes absent), and they have only 
been edited to make them better readable within web pages and to fix the few material errors that have been found. The order in which they are presented is chronological.

The role of measurement in the development of industrial automation, by S.S. Carlisle [Acta IMEKO 1967, Proceedings of the $4^{\text {th }}$ International Measurement Congress, 1967, Warsaw, vol. 1, pp. 37-50], is far-sighted paper that sets a frame on the role of measurement and control as critical tools to support the automation of manufacturing processes. In this perspective the Author discusses three main requirements for measurement, i.e., to identify where automation can be most profitably used; to investigate individual process behaviors and hence to formulate process control strategies; and finally to perform quality control of products. Also with the support of several practical examples, with interesting mentions to the growing importance of digital computer and pattern recognition techniques, the Author proposes a systemic vision, in which measurement is convincingly intended as a communication enabler among the different subjects involved in industrial innovation processes: control engineers, process and product engineers, managers, and end users.

Fundamental concepts of measurement, by L. Finkelstein [Acta IMEKO 1973, Proceedings of the $6^{\text {th }}$ Congress of the International Measurement Confederation, "Measurement and instrumentation”, 17-23.6.1973, Dresden, vol. 1, pp. 11-27], proposes a clear, synthetic presentation of some basics of measurement science. While in the last forty years many things have changed in the technological aspects of measurement (so that, in particular, what the Author calls "associative measurement" is much more widespread than one might suppose from the short mention that is devoted here), the conceptual framework that follows is an excellent entry point for the reader interested in the epistemological and logical foundations of measurement.

The measurement chain and validation of experimental measurements, by R.J. Moffat [Acta IMEKO 1973, Proceedings of the $6^{\text {th }}$ Congress of the International Measurement Confederation, "Measurement and instrumentation", 17-23.6.1973, Dresden, vol. 1, pp. 45-53], witnesses the sophisticated discussion that, well before the publication of the Guide to the Expression of Uncertainty in Measurement (GUM), was active in the measurement science community around the subject of error and uncertainty, and its consequences on the structure of the measuring process and the way it is performed. Some of the ideas presented here, and particularly the hypothesis that what the GUM calls the standard uncertainty admits multiple methods of computation depending on the aim of the process, are still delicate, open issues today.

Problems in theory of measurement today, by L. Gonella [Acta IMEKO 1979, Proceedings of the $8^{\text {th }}$ IMEKO Congress of the International Measurement Confederation, "Measurement for progress in science and technology", 21-27.5.1979, Moskow, vol. 1, pp. 103-110], challenges, in an informed and convincing way, some basic presuppositions of the "classical" theory of measurement, particularly highlighting the biases induced by an often acritical adoption of the geometric paradigm, and consequently of real numbers, in measurement.

Theoretical, physical and metrological problems of further development of measurement techniques and instrumentation in science and technology, by D. Hofmann and Yu. V. Tarbeyev [Acta IMEKO 1979, Proceedings of the $8^{\text {th }}$ IMEKO Congress of the International
Measurement Confederation, "Measurement for progress in science and technology", 21-27.5.1979, Moskow, vol. 3, pp. 607-626], lists a number of issues of measurement science and technology, thus interestingly witnessing their state in the second half of the $20^{\text {th }}$ century.

Measurement and meaningfulness, by J. van Brakel [Acta IMEKO 1985, Proceedings of the $10^{\text {th }}$ World Congress of the International Measurement Confederation, "New measurement technology to serve mankind", 22-26.4.1985, Prague, pp. 319$333]$, is a learned paper on a key concept in measurement, i.e., the meaningfulness of statements reporting measurement results, whose connections, among the others, to scale invariance, conditions for derived measurement, dimensional analysis, and definition of measurement standards are clearly presented and discussed also by means of several examples.

Structured instrument design, by P.H. Sydenham [Acta IMEKO 1988, Proceedings of the $11^{\text {th }}$ Triennial World Congress of the International Measurement Confederation (IMEKO), "Instrumentation for the 21 st century", 16-21.10.1988, Houston, pp. 271-275], proposes an overview on the delicate subject of the possibility of automatic design and configuration of measuring systems ("CAD of instruments", or "instrument CAE"), in a well-conceived scenario of measuring systems intended as an important class of information machines. Despite in those early days of computer-aided processes the position, sometimes made into a research plan, was not unusual that the creative activity of development can be fully automatized (as in the case of the efforts towards universal software code generators), the Author is well aware of the importance not only of formal algorithms, but also of heuristics, "rules of thumb", and even subjective judgement, critical source of complexity for the purported target.

Impact of modern instrumentation on the system of basic concepts in metrology, by J.M. Jaworski, J. Bek, and A. J. Fiok [Acta IMEKO 1988, Proceedings of the Proceedings of the $11^{\text {th }}$ Triennial World Congress of the International Measurement Confederation (IMEKO), "Instrumentation for the $21^{\text {st }}$ century", 16-21.10.1988, Houston, pp. 125-134], offers several analytical arguments on the practical importance of rethinking the basics of measurement science as a concept system, in response to the critical increase of complexity of measurement problems in both spatial and temporal dimensions, i.e., relating to multivariate, dynamic quantities. The authors significantly emphasize the role of models and of pragmatics, including the costs of designing and performing the process.

Intelligent instrumentation: a quality challenge, by H.J. Kohoutek [Acta IMEKO 1988, Proceedings of the Proceedings of the $11^{\text {th }}$ Triennial World Congress of the International Measurement Confederation (IMEKO), "Instrumentation for the $21^{\text {st }}$ century", 16-21.10.1988, Houston, pp. 337-345], presents a systematic analysis of the consequences of the adoption of artificial intelligence techniques in measuring systems and the related open issues. The author emphasizes that the growing importance of software components offers new opportunities for the development of flexible, "intelligent" instrumentation, but at the same time poses new problems in the metrological assurance of their behavior.

Our hope is that, together with the pride and the curiosity that this small sample can produce on IMEKO and its history, the reader can find here new reasons of interest for measurement science and its fundamental role for our society. 